\title{
Multivalued SK-contractions with respect to $b$-generalized pseudodistances
}

\section{Moosa Gabeleh and Robert Plebaniak ${ }^{2 *}$}

\section{${ }^{*}$ Correspondence:}

robpleb@math.uni.lodz.pl

${ }^{2}$ Department of Nonlinear Analysis,

University of Łódź, Banacha 22,

Łódź, 90-238, Poland

Full list of author information is

available at the end of the article

\begin{abstract}
A new class of multivalued non-self-mappings, called SK-contractions with respect to $b$-generalized pseudodistances, is introduced and used to investigate the existence of best proximity points by using an appropriate geometric property. Some new fixed point results in $b$-metric spaces are also obtained. Examples are given to support the usability of our main results.
\end{abstract}

MSC: 47H10; 47H09; 46B20

Keywords: best proximity point; fixed point; SK-contraction; b-generalized pseudodistances

\section{Introduction}

Let $A$ and $B$ be two nonempty subsets of a metric space $X$. A non-self-mapping $T: A \rightarrow B$ is said to be a contraction if there exists a constant $r \in[0,1)$, such that $d(T x, T y) \leq r d(x, y)$, for all $x, y \in A$. The well-known Banach contraction principle states that if $A$ is a complete subset of $X$ and $T$ is a contraction self-mapping, then the fixed point equation $T x=x$ has exactly one solution.

The Banach contraction principle is a very important tool in nonlinear analysis and there are many extensions of this principle; see, e.g., [1] and the references therein.

Let $(X, d)$ be a metric space. A self-mapping $T: X \rightarrow X$ is called a Kannan mapping if there exists $\alpha \in\left[0, \frac{1}{2}\right)$ such that

$$
d(T x, T y) \leq \alpha[d(x, T x)+d(y, T y)]
$$

for all $x, y \in X$. We know that if $X$ is a complete metric space, every Kannan self-mapping defined on $X$ has a unique fixed point [2]. Note that the notion of contraction mappings and Kannan mappings are independent. That is, there exists a contraction mapping which is not a Kannan, and a Kannan mapping which is not a contraction. Therefore, we cannot compare these two classes of mappings directly. However, the Banach and Kannan fixed point theorems can be unified (for details see [3-5]).

Kikkawa and Suzuki in [6], established the following fixed point theorem, which is an extension of the Kannan fixed point theorem.

C 2015 Gabeleh and Plebaniak; licensee Springer. This is an Open Access article distributed under the terms of the Creative Commons Attribution License (http://creativecommons.org/licenses/by/4.0), which permits unrestricted use, distribution, and reproduction in any medium, provided the original work is properly credited. 
Theorem 1.1 ([6]) Define a non-increasing function $\varphi$ from $[0,1)$ into $\left(\frac{1}{2}, 1\right]$ by

$$
\varphi(r)= \begin{cases}1 & \text { if } 0 \leq r<\frac{1}{\sqrt{2}} \\ \frac{1}{1+r} & \text { if } \frac{1}{\sqrt{2}} \leq r<1\end{cases}
$$

Let $(X, d)$ be a complete metric space and let $T$ be a self-mapping on $X$. Let $\alpha \in\left[0, \frac{1}{2}\right)$ and put $r:=\frac{\alpha}{1-\alpha} \in[0,1)$. Assume that

$$
\varphi(r) d(x, T x) \leq d(x, y) \quad \text { implies } \quad d(T x, T y) \leq \alpha[d(x, T x)+d(y, T y)]
$$

for all $x, y \in X$. Then $T$ has a unique fixed point $z$ and $\lim _{n} T^{n} x=z$ holds for every $x \in X$.

It is interesting to note that the function $\varphi(r)$ defined in Theorem 1.1 is the best constant for every $r$ (see Theorem 2.4 of [6]).

The multivalued version of Theorem 1.1 was presented in [7] as below.

Theorem 1.2 (Damjanovic and Doric [7]) Define a non-increasing function $\varphi(r)$ from $[0,1)$ into $(0,1]$ by

$$
\varphi(r)= \begin{cases}1 & \text { if } 0 \leq r<\frac{\sqrt{5}-1}{2} \\ 1-r & \text { if } \frac{\sqrt{5}-1}{2} \leq r<1\end{cases}
$$

Let $(X, d)$ be a complete metric space and let $T$ be a mapping from $X$ into $\mathcal{C B}(X)$, where $\mathcal{C B}(X)$ denotes the family of all nonempty, bounded and closed subsets of $X$. Assume that

$$
\varphi(r) d(x, T x) \leq d(x, y) \quad \text { implies } \quad \mathcal{H}(T x, T y) \leq r \max \{D(x, T x), D(y, T y)\}
$$

for all $x, y \in X$, where $D(x, A):=\inf \{d(x, y): y \in A\}$ for $x \in X$ and $A \subset X$. Then there exists $z \in X$ such that $z \in T z$.

Now, let $(A, B)$ be a nonempty pair of subsets of a metric space $(X, d)$ and let $T: A \rightarrow 2^{B}$ be a multivalued non-self-mapping. Then for each $x \in A$ we have $\mathcal{D}(x, T x) \geq \operatorname{dist}(A, B)$, where $\operatorname{dist}(A, B):=\inf \{d(x, y):(x, y) \in A \times B\}$ and $\mathcal{D}(x, T x):=\operatorname{dist}(\{x\}, T x)$. So, it is quite natural to seek an approximate solution $x \in A$ that is optimal in the sense that the distance $\mathcal{D}(x, T x)$ with respect to $\mathcal{D}$ is minimum. As the minimality of the value $\mathcal{D}(x, T x)$ connotes the highest closeness between the elements $x$ and $T x$, one attempts to determine an element $x$ for which $\mathcal{D}(x, T x)$ assumes the least possible value $\operatorname{dist}(A, B)$. Such an optimal solution $x$ for which $\mathcal{D}(x, T x)=\operatorname{dist}(A, B)$ is called a best proximity point of the multivalued non-self-mapping $T$. The existence of best proximity points for multivalued non-selfmappings was first studied in [8] for multivalued nonexpansive non-self-mappings in hyperconvex metric spaces and in Hilbert spaces (see also [9-13] for different approaches to the same problem).

The main purpose of this article is to elicit a best proximity point theorem for a new class of multivalued non-self-mappings in the setting of $b$-metric spaces. Our results improve and extend the main results in [6,7]. 


\section{Preliminaries}

Let $A$ and $B$ be two nonempty subsets of a metric space $(X, d)$. When we say that a pair $(A, B)$ satisfies a special property, we mean that both $A$ and $B$ satisfy the mentioned property. We will use the following notations:

$$
\begin{aligned}
& \forall_{a \in A}\left\{\mathcal{D}^{*}(a, B)=\mathcal{D}(a, B)-\operatorname{dist}(A, B)\right\}, \\
& \forall_{A, B \in \mathcal{C} \mathcal{B}(X)}\left\{\mathcal{H}(A, B)=\max \left\{\sup _{x \in A} \mathcal{D}(x, B), \sup _{y \in B} \mathcal{D}(y, A)\right\}\right\}, \\
& A_{0}=\{x \in A: d(x, y)=\operatorname{dist}(A, B), \text { for some } y \in B\}, \\
& B_{0}=\{y \in B: d(x, y)=\operatorname{dist}(A, B), \text { for some } x \in A\} .
\end{aligned}
$$

It is easy to see that if $(A, B)$ is a nonempty weakly compact pair in a Banach space $X$, then $\left(A_{0}, B_{0}\right)$ is a pair of nonempty subsets of $X$.

Definition 2.1 Let $(A, B)$ be a pair of nonempty subsets of a metric space $(X, d)$ with $A_{0} \neq \varnothing$.

(I) [14] The pair $(A, B)$ is said to have the P-property if and only if

$$
\left\{\begin{array}{l}
d\left(x_{1}, y_{1}\right)=\operatorname{dist}(A, B), \\
d\left(x_{2}, y_{2}\right)=\operatorname{dist}(A, B)
\end{array} \quad \Rightarrow \quad d\left(x_{1}, x_{2}\right)=d\left(y_{1}, y_{2}\right),\right.
$$

where $x_{1}, x_{2} \in A_{0}$ and $y_{1}, y_{2} \in B_{0}$.

(II) [15] The pair $(A, B)$ is said to have the WP-property if and only if

$$
\left\{\begin{array}{l}
d\left(x_{1}, y_{1}\right)=\operatorname{dist}(A, B), \\
d\left(x_{2}, y_{2}\right)=\operatorname{dist}(A, B)
\end{array} \quad \Rightarrow \quad d\left(x_{1}, x_{2}\right) \leq d\left(y_{1}, y_{2}\right),\right.
$$

where $x_{1}, x_{2} \in A_{0}$ and $y_{1}, y_{2} \in B_{0}$.

The notion of the WP-property notion is weaker than the notion of the P-property.

Example 2.1 ([14]) Let $(A, B)$ be a nonempty, closed, and convex pair of subsets of a Hilbert space $\mathbb{H}$. Then $(A, B)$ satisfies the WP-property.

Example 2.2 Let $(A, B)$ be a nonempty pair of subsets of a metric space $(X, d)$ such that $A_{0} \neq \emptyset$ and $\operatorname{dist}(A, B)=0$. Then $(A, B)$ has the WP-property.

Example 2.3 ([16]) Let $(A, B)$ be a nonempty bounded, closed and convex pair of subsets of a uniformly convex Banach space $X$. Then $(A, B)$ has the WP-property.

Example 2.4 Consider $X:=\mathbb{R}$ with the usual metric. Suppose that

$$
A:=[1,2] \quad \text { and } \quad B:=\{-1,0,3\} \text {. }
$$

Then we have $\operatorname{dist}(A, B)=1$ and $A_{0}=\{1,2\}, B_{0}:=\{0,3\}$. If $\left(x_{1}, x_{2}\right)=(1,2)$ and $\left(y_{1}, y_{2}\right)=(0,3)$, then

$$
d\left(x_{1}, y_{1}\right)=d\left(x_{2}, y_{2}\right)=\operatorname{dist}(A, B) \quad \text { and } \quad d\left(x_{1}, x_{2}\right)<d\left(y_{1}, y_{2}\right) \text {, }
$$


from which one deduces that $(A, B)$ has the WP-property. Note that $(B, A)$ does not have the WP-property and so $(A, B)$ does not have the P-property. We mention that in [17] the authors studied the existence of some nonlinear programming problems by using the geometric notion of the WP-property.

The notion of $b$-metric space was introduced by Czerwik [18] as below.

Definition 2.2 ([18]) Let $X$ be a nonempty set and $s \geq 1$ be a given real number. A function $d: X \times X \rightarrow[0, \infty)$ is $b$-metric if the following three conditions are satisfied:

$\left(\mathrm{d}_{1}\right) \forall_{x, y \in X}\{d(x, y)=0 \Leftrightarrow x=y\}$;

$\left(\mathrm{d}_{2}\right) \forall_{x, y \in X}\{d(x, y)=d(y, x)\}$;

$\left(\mathrm{d}_{3}\right) \forall_{x, y, z \in X}\{d(x, z) \leq s[d(x, y)+d(y, z)]\}$.

If $d$ is a $b$-metric on $X$ (with constant $s \geq 1$ ), then the pair $(X, d)$ is called a $b$-metric space. Note that every metric space is a $b$-metric space. Throughout this paper, we assume that the $b$-metric $d: X \times X \rightarrow[0, \infty)$ is continuous on $X^{2}$. For more precise information as regards $b$-metric spaces and best proximity point results we can see [19-27].

One can refer to [28] for the interesting results concerning fixed point theorems in $b$-metric spaces.

Definition 2.3 ([29]) Let $X$ be a $b$-metric space (with constant $s \geq 1$ ). The map $J: X \times X \rightarrow$ $[0, \infty)$ is said to be a $b$-generalized pseudodistance on $X$ if the following two conditions hold:

(J1) $\forall_{x, y, z \in X}\{J(x, z) \leq s[J(x, y)+J(y, z)]\}$;

(J2) for any sequences $\left(x_{m}: m \in \mathbb{N}\right)$ and $\left(y_{m}: m \in \mathbb{N}\right)$ in $X$ such that

$$
\lim _{n \rightarrow \infty} \sup _{m>n} J\left(x_{n}, x_{m}\right)=0
$$

and

$$
\lim _{m \rightarrow \infty} J\left(x_{m}, y_{m}\right)=0
$$

we have

$$
\lim _{m \rightarrow \infty} d\left(x_{m}, y_{m}\right)=0 .
$$

Remark 2.4 If $(X, d)$ is a $b$-metric space (with $s \geq 1$ ), then the $b$-metric $d: X \times X \rightarrow[0, \infty)$ is a $b$-generalized pseudodistance on $X$. However, there exists a $b$-generalized pseudodistance on $X$ which is not a $b$-metric (for details see Example 4.1 of [29]).

Remark 2.5 From (J1) and (J2) it follows that if $x \neq y, x, y \in X$, then

$$
J(x, y)>0 \vee J(y, x)>0 .
$$

It is worth noticing that in fixed point theory, on defining contractions, many authors replaced the metric by some more general mapping. In the literature there exist many examples of different distances. Now we recall some of them. 
Let $X$ be a metric space with metric $d$. Then a function $p$ from $X \times X$ into $\mathbb{R}_{+}$is called a $w$-distance on $X$ if it satisfies the following:

(p1) $p(x, y) \leq p(x, z)+p(z, y)$ for all $x, y, z \in X$;

(p2) $p$ is lower semicontinuous in its second variable;

(p3) for each $\varepsilon>0$, there exists $\delta>0$ such that $p(z, x) \leq \delta$ and $p(z, y) \leq \delta$ imply $p(x, y) \leq \varepsilon$.

The metric $d$ is a $w$-distance on $X$. The concept of $w$-distance was first introduced by Kada et al. [30].

Let $X$ be a subset of a Banach space and let $\left\{T(t): t \in \mathbb{R}_{+}\right\}$be a strongly continuous semigroup of nonexpansive mappings on $X$, i.e.,

(sg1) for each $t \in \mathbb{R}_{+}, T(t)$ is a nonexpansive mapping on $X$;

(sg2) $T(0) x=x$, for all $x \in X$;

(sg3) $T(s+t)=T(s) \circ T(t)$ for all $s, t \in \mathbb{R}_{+}$;

(sg4) for each $x \in X$, the mapping $T(x)$ from $\mathbb{R}_{+}$into $X$ is continuous.

In [31], Tataru introduced the distance

$$
p(x, y)=\inf \left\{t+\|T(t) x-y\|: t \in \mathbb{R}_{+}\right\},
$$

for all $x, y \in X$.

Let $X$ be a metric space with metric $d$. Then a function $p$ from $X \times X$ into $\mathbb{R}_{+}$is called a $\tau$-distance on $X$ (introduced by Suzuki [32]) if there exists a function $\eta: X \times \mathbb{R}_{+}$into $\mathbb{R}_{+}$ and the following are satisfied:

(S1) $\forall_{x, y, z \in X}\{p(x, z) \leq p(x, y)+p(y, z)\}$

(S2) $\forall_{x \in X} \forall_{t>0}\{\eta(x, 0)=0 \wedge \eta(x, t) \geq t\}$ and $\eta$ is concave and continuous in its second variable;

$$
\begin{array}{r}
\left\{\lim _{n \rightarrow \infty} x_{n}=x \wedge \lim _{n \rightarrow \infty} \sup _{m \geq n} \eta\left(z_{n}, p\left(z_{n}, x_{m}\right)\right)=0\right\} \\
\Rightarrow \quad\left\{\forall_{w \in X}\left\{p(w, x) \leq \lim \inf _{n \rightarrow \infty} p\left(w, x_{n}\right)\right\}\right\} ;
\end{array}
$$

(S4) $\left\{\lim _{n \rightarrow \infty} \sup _{m \geq n} p\left(x_{n}, y_{m}\right)=0 \wedge \lim _{n \rightarrow \infty} \eta\left(x_{n}, t_{n}\right)=0\right\} \Rightarrow\left\{\lim _{n \rightarrow \infty} \eta\left(y_{n}, t_{n}\right)=0\right\}$;

(S5) $\left\{\lim _{n \rightarrow \infty} \eta\left(z_{n}, p\left(z_{n}, x_{n}\right)\right)=0 \wedge \lim _{n \rightarrow \infty} \eta\left(z_{n}, p\left(z_{n}, y_{n}\right)\right)=0\right\} \Rightarrow\left\{\lim _{n \rightarrow \infty} d\left(x_{n}, y_{n}\right)=0\right\}$.

In 2006, Lin and $\mathrm{Du}$ [33] introduced the following concept of a $\tau$-function.

Let $X$ be a metric space with metric $d$. A map $p$ from $X \times X$ into $\mathbb{R}_{+}$is called a $\tau$-function on $X$ if the following conditions hold:

(L1) $p(x, y) \leq p(x, z)+p(z, y)$ for all $x, y, z \in X$;

(L2) if $x \in X$ and $\left\{y_{n}\right\}$ in $X$ with $\lim _{n \rightarrow \infty} y_{n}=y$ and $p\left(x, y_{n}\right) \leq M$ for some $M=M(x)>0$, then $p(x, y) \leq M$;

(L3) for any sequence $\left\{x_{n}\right\}$ in $X$ with $\lim _{n \rightarrow \infty} \sup \left\{p\left(x_{n}, x_{m}\right): m>n\right\}=0$, if there exists a sequence $\left\{y_{n}\right\}$ in $X$ such that $\lim _{n \rightarrow \infty} p\left(x_{n}, y_{n}\right)=0$, then $\lim _{n \rightarrow \infty} d\left(x_{n}, y_{n}\right)=0$;

(L4) for $x, y, z \in X, p(x, y)=0$, and $p(x, z)=0$ imply that $y=z$.

In 1985, Valyi [34] introduced and used in uniform spaces the new concept of distance which in our conventions we will call the Valyi distance.

Let $X$ be a metric space with metric $d$. A map $p$ from $X \times X$ into $\mathbb{R}_{+}$is called a distance of Valyi on $X$ if the following conditions hold: 
(V1) $p(x, y) \leq p(x, z)+p(z, y)$ for all $x, y, z \in X$;

(V2) $p$ is lsc in its second variable;

(V3) $\forall_{x, y \in X}\{p(x, y) \geq 0 \wedge[p(x, y)=0 \Leftrightarrow x=y]\}$;

(V4) $\forall_{\varepsilon>0} \exists_{\delta>0} \forall_{x, y \in X}\{p(x, y)<\delta \Rightarrow d(x, y)<\varepsilon\}$.

\section{Remark 2.6}

(i) Let $p$ be a $w$-distance on a metric space $X$. Then $p$ is also a $\tau$-distance on $X$ (see Proposition 1 in [32]).

(ii) Let $\left\{T(t): t \in \mathbb{R}_{+}\right\}$be a strongly continuous semigroup of nonexpansive mappings on a subset $X$ of a Banach space. Then the Tataru distance $p$ on $X$ is also a $\tau$-distance on $X$ (see Proposition 2 in [32]).

(iii) Let $p$ be a $w$-distance on $X$. Then $p$ is a $\tau$-function on $X$ (see Remark 2.1 in [33]).

(iv) In the literature there are no studies concerning relations between Valyi distances [34] and $\tau$-distances [32] and $\tau$-functions [33].

Remark 2.7 ([35]) Let $X$ be a $b$-metric space (with $s=1$ ), i.e. $X$ be a metric space with metric $d$. Then

(i) if $p$ is a $\tau$-distance, then $p$ is a generalized pseudodistance;

(ii) if $p$ is a $\tau$-function, then $p$ is a generalized pseudodistance;

(iii) if $p$ is a Valyi distance, then $p$ is a generalized pseudodistance;

(iv) there exists a generalized pseudodistance which is not a $\tau$-distance;

(v) there exists a generalized pseudodistance which is not $\tau$-function;

(vi) there exists a generalized pseudodistance which is not a Valyi distance.

After this short introduction concerning the distances that were used in fixed point theory, by using the notion of $b$-generalized pseudodistance on a $b$-metric space $X$, we can define the $\mathcal{H}^{J}$ Hausdorff distance as below.

Definition 2.8 Let $X$ be a $b$-metric space (with $s \geq 1$ ) and let the map $J: X \times X \rightarrow[0, \infty$ ) be a $b$-generalized pseudodistance on $X$. Let $\forall_{u \in X} \forall_{V \in \mathcal{C} \mathcal{B}(X)}\left\{(u, V)=\inf _{v \in V} J(u, v)\right\}$. Define $\mathcal{H}^{J}: \mathcal{C B}(X) \times \mathcal{C B}(X) \rightarrow[0, \infty)$ by

$$
\forall_{A, B \in \mathcal{C} \mathcal{B}(X)}\left\{\mathcal{H}^{J}(A, B)=\max \left\{\sup _{u \in A} J(u, B), \sup _{v \in B} J(v, A)\right\}\right\} .
$$

Similarly, the following definitions and notations can be constructed in $b$-metric spaces equipped with a $b$-generalized pseudodistance.

Let $(X, d)$ be a $b$-metric space (with $s \geq 1)$ and let $(A, B)$ be a pair of subsets of $X$ and let the map $J: X \times X \rightarrow[0, \infty)$ be a $b$-generalized pseudodistance on $X$. We set

$$
\begin{aligned}
& A_{0}:=\{x \in A: J(x, y)=\operatorname{dist}(A, B), \text { for some } y \in B\} ; \\
& B_{0}:=\{y \in B: J(x, y)=\operatorname{dist}(A, B), \text { for some } x \in A\} ; \\
& J^{*}(a, B)=\frac{1}{S} J(a, B)-\operatorname{dist}(A, B), \quad \text { for } a \in A .
\end{aligned}
$$

Definition 2.9 Let $X$ be a $b$-metric space (with $s \geq 1$ ) and let the map $J: X \times X \rightarrow[0, \infty)$ be a $b$-generalized pseudodistance on $X$. Let $(A, B)$ be a pair of nonempty subset of $X$ with $A_{0} \neq \emptyset$. 
(I) The pair $(A, B)$ is said to have the $\mathrm{WP}^{J}$-property if and only if

$$
\begin{aligned}
& \left\{\left[J\left(x_{1}, y_{1}\right)=\operatorname{dist}(A, B)\right] \wedge\left[J\left(x_{2}, y_{2}\right)=\operatorname{dist}(A, B)\right]\right\} \\
& \quad \Rightarrow \quad\left\{J\left(x_{1}, x_{2}\right) \leq J\left(y_{1}, y_{2}\right)\right\}
\end{aligned}
$$

where $x_{1}, x_{2} \in A_{0}$ and $y_{1}, y_{2} \in B_{0}$.

(II) We say that the $b$-generalized pseudodistance $J$ is associated with the pair $(A, B)$ if for any sequences $\left(x_{m}: m \in \mathbb{N}\right)$ and $\left(y_{m}: m \in \mathbb{N}\right)$ in $X$ such that $\lim _{m \rightarrow \infty} x_{m}=x$; $\lim _{m \rightarrow \infty} y_{m}=y$; and

$$
\forall_{m \in \mathbb{N}}\left\{J\left(x_{m}, y_{m-1}\right)=\operatorname{dist}(A, B)\right\}
$$

then $d(x, y)=\operatorname{dist}(A, B)$.

We mention that for a $b$-metric space $(X, d)$ if we put $J=d$, then the map $d$ is associated with each pair $(A, B)$, where $(A, B)$ is a nonempty pair in $X$ because of the continuity of $d$.

Definition 2.10 Let $\tau$ be a topological vector space. Let $X$ be a certain space and $(A, B)$ be a nonempty pair of subsets of $X$. The multivalued non-self-mapping $T: A \rightarrow 2^{B}$ is called closed whenever $\left(x_{m}: m \in \mathbb{N}\right)$ is a sequence in $A$ converging to $x \in A$ and $\left(y_{m}: m \in \mathbb{N}\right)$ is a sequence in $B$ satisfying the condition $\forall_{m \in \mathbb{N}}\left\{y_{m} \in T\left(x_{m}\right)\right\}$ and converging to $y \in B$, then $y \in T(x)$.

The following lemma will be used in the sequel.

Lemma 2.11 ([29]) Let $X$ be a complete b-metric space (with $s \geq 1$ ) equipped with the $b$-generalized pseudodistance $J$ and let the sequence $\left(x_{m}: m \in\{0\} \cup \mathbb{N}\right)$ satisfy

$$
\lim _{n \rightarrow \infty} \sup _{m>n} J\left(x_{n}, x_{m}\right)=0
$$

Then $\left(x_{m}: m \in\{0\} \cup \mathbb{N}\right)$ is a Cauchy sequence on $X$.

\section{Main results}

We begin our main results of this paper with the following notion.

Definition 3.1 Define the strictly decreasing function $\zeta:\left[0, \frac{1}{2}\right) \rightarrow\left(\frac{1}{2}, 1\right]$ by

$$
\zeta(\alpha)=1-\alpha
$$

Let $X$ be a $b$-metric space (with $s \geq 1$ ) and let the map $J: X \times X \rightarrow[0, \infty$ ) be a $b$-generalized pseudodistance on $X$. Let $(A, B)$ be a pair of nonempty of subsets of $X$. A multivalued nonself-mapping $T: A \rightarrow 2^{B}$ is said to be an SK-contraction with respect to $b$-generalized pseudodistances provided that

$$
\begin{aligned}
& \exists_{\alpha \in\left[0, \frac{1}{2}\right)}, \forall_{x, y \in A}\left\{\left\{\frac{\zeta(\alpha)}{s} J^{*}(x, T(x)) \leq J(x, y)\right\}\right. \\
& \left.\Rightarrow\left\{s \mathcal{H}^{J}(T(x), T(y)) \leq \alpha\left[J^{*}(x, T x)+J^{*}(y, T y)\right]\right\}\right\} .
\end{aligned}
$$


We now state the main result of this paper.

Theorem 3.2 Let $X$ be a complete b-metric space (with $s \geq 1$ ) and let the map J:X $\times X \rightarrow$ $[0, \infty)$ be a b-generalized pseudodistance on $X$. Let $(A, B)$ be a pair of nonempty closed subsets of $X$ with $A_{0} \neq \emptyset$ and such that $(A, B)$ has the WP'-property and $J$ is associated with $(A, B)$. Let $T: A \rightarrow 2^{B}$ be a closed multivalued non-self-mapping which is an SK-contraction with respect to b-generalized pseudodistances. If $T(x) \in \mathcal{C B}(X)$ for all $x \in A$, and $T(x) \subset B_{0}$ for each $x \in A_{0}$, then $T$ has a best proximity point in $A$.

Proof Take a real number $\beta$ with $0 \leq \alpha<\beta<\frac{1}{2}$. Let $x_{0} \in A_{0}$ and $y_{0} \in T x_{0}$. Since $T x_{0} \subseteq B_{0}$, there exists $x_{1} \in A_{0}$ such that $J\left(x_{1}, y_{0}\right)=\operatorname{dist}(A, B)$. We have

$$
J\left(x_{0}, T x_{0}\right) \leq d\left(x_{0}, y_{0}\right) \leq s\left[J\left(x_{0}, x_{1}\right)+J\left(x_{1}, y_{0}\right)\right]
$$

and so

$$
J^{*}\left(x_{0}, T x_{0}\right)=\frac{1}{s} J\left(x_{0}, T x_{0}\right)-\operatorname{dist}(A, B) \leq \frac{1}{s} s\left[J\left(x_{0}, x_{1}\right)+J\left(x_{1}, y_{0}\right)\right]-\operatorname{dist}(A, B)=J\left(x_{0}, x_{1}\right) .
$$

Since $\frac{\zeta(\alpha)}{s} \leq 1$, we obtain

$$
\frac{\zeta(\alpha)}{s} J^{*}\left(x_{0}, T x_{0}\right) \leq J\left(x_{0}, x_{1}\right) .
$$

It now follows from the fact that $T$ is an SK-contraction with respect to $b$-generalized pseudodistances that

$$
\begin{aligned}
s J\left(y_{0}, T x_{1}\right) & \leq s \mathcal{H}^{J}\left(T x_{0}, T x_{1}\right) \leq \alpha\left[J^{*}\left(x_{0}, T x_{0}\right)+J^{*}\left(x_{1}, T x_{1}\right)\right] \\
& <\beta\left[J^{*}\left(x_{0}, T x_{0}\right)+J^{*}\left(x_{1}, T x_{1}\right)\right] \\
& =\beta\left[\frac{1}{s}\left(J\left(x_{0}, T x_{0}\right)+J\left(x_{1}, T x_{1}\right)\right)-2 \operatorname{dist}(A, B)\right] .
\end{aligned}
$$

Consider $y_{1} \in T x_{1}$ such that $s J\left(y_{0}, y_{1}\right) \leq \beta\left[\frac{1}{s}\left(J\left(x_{0}, T x_{0}\right)+J\left(x_{1}, T x_{1}\right)\right)-2 \operatorname{dist}(A, B)\right]$. Then

$$
\begin{aligned}
J\left(y_{0}, y_{1}\right) & \leq \frac{\beta}{s}\left[\frac{1}{s}\left(J\left(x_{0}, y_{0}\right)+J\left(x_{1}, y_{1}\right)\right)-2 \operatorname{dist}(A, B)\right] \\
& \leq \frac{\beta}{s}\left[\frac{1}{s}\left[s\left(J\left(x_{0}, x_{1}\right)+J\left(x_{1}, y_{0}\right)\right)+s\left(J\left(x_{1}, y_{0}\right)+J\left(y_{0}, y_{1}\right)\right)\right]-2 \operatorname{dist}(A, B)\right] \\
& =\frac{\beta}{s}\left[J\left(x_{0}, x_{1}\right)+J\left(y_{0}, y_{1}\right)\right],
\end{aligned}
$$

which implies that

$$
J\left(y_{0}, y_{1}\right) \leq \frac{\beta}{s-\beta} J\left(x_{0}, x_{1}\right) .
$$

Again, since $T x_{1} \subseteq B_{0}$ and $y_{1} \in T x_{1}$, there exists $x_{2} \in A_{0}$ such that $J\left(x_{2}, y_{1}\right)=\operatorname{dist}(A, B)$. Also,

$$
J\left(x_{1}, T x_{1}\right) \leq J\left(x_{1}, y_{1}\right) \leq s\left[J\left(x_{1}, x_{2}\right)+J\left(x_{2}, y_{1}\right)\right],
$$


from which one concludes that

$$
\begin{aligned}
J^{*}\left(x_{1}, T x_{1}\right) & =\frac{1}{s} J\left(x_{1}, T x_{1}\right)-\operatorname{dist}(A, B) \\
& \leq \frac{1}{s} s\left[J\left(x_{1}, x_{2}\right)+J\left(x_{2}, y_{1}\right)\right]-\operatorname{dist}(A, B)=J\left(x_{1}, x_{2}\right),
\end{aligned}
$$

and so

$$
\frac{\zeta(\alpha)}{s} J^{*}\left(x_{1}, T x_{1}\right) \leq J\left(x_{1}, x_{2}\right) .
$$

Thus

$$
\begin{aligned}
s J\left(y_{1}, T x_{2}\right) & \leq s \mathcal{H}^{J}\left(T x_{1}, T x_{2}\right) \leq \alpha\left[J^{*}\left(x_{1}, T x_{1}\right)+J^{*}\left(x_{2}, T x_{2}\right)\right] \\
& \leq \beta\left[\frac{1}{s}\left(J\left(x_{1}, T x_{1}\right)+J\left(x_{2}, T x_{2}\right)\right)-2 \operatorname{dist}(A, B)\right] .
\end{aligned}
$$

Therefore, there exists $y_{2} \in T x_{2}$ such that

$$
\begin{aligned}
s J\left(y_{1}, y_{2}\right) & \leq \beta\left[\frac{1}{s}\left(J\left(x_{1}, T x_{1}\right)+J\left(x_{2}, T x_{2}\right)\right)-2 \operatorname{dist}(A, B)\right] \\
& \leq \beta\left[\frac{1}{s}\left(J\left(x_{1}, y_{1}\right)+J\left(x_{2}, y_{2}\right)\right)-2 \operatorname{dist}(A, B)\right] \\
& \leq \beta\left[\frac{1}{s}\left[s\left(J\left(x_{1}, x_{2}\right)+J\left(x_{2}, y_{1}\right)\right)+s\left(J\left(x_{2}, y_{1}\right)+J\left(y_{1}, y_{2}\right)\right)\right]-2 \operatorname{dist}(A, B)\right] \\
& =\beta\left[J\left(x_{1}, x_{2}\right)+J\left(y_{1}, y_{2}\right)\right] .
\end{aligned}
$$

We now have

$$
J\left(y_{1}, y_{2}\right) \leq \frac{\beta}{s-\beta} J\left(x_{1}, x_{2}\right)
$$

Continuing this process, by induction we can find sequences $\left(x_{m}: m \in\{0\} \cup \mathbb{N}\right)$ and $\left(y_{m}\right.$ : $m \in\{0\} \cup \mathbb{N}$ ) such that

$$
\begin{aligned}
& \forall_{m \in\{0\} \cup \mathbb{N}}\left\{x_{m} \in A_{0} \wedge y_{m} \in B_{0}\right\}, \\
& \forall_{m \in\{0\} \cup \mathbb{N}}\left\{y_{m} \in T\left(x_{m}\right)\right\}, \\
& \forall_{m \in \mathbb{N}}\left\{J\left(x_{m}, y_{m-1}\right)=\operatorname{dist}(A, B)\right\}
\end{aligned}
$$

and

$$
\forall_{m \in \mathbb{N}}\left\{J\left(y_{m-1}, y_{m}\right) \leq \frac{\beta}{s-\beta} J\left(x_{m-1}, x_{m}\right)\right\} .
$$

We now have $\forall_{m \in \mathbb{N}}\left\{J\left(x_{m}, y_{m-1}\right)=\operatorname{dist}(A, B) \wedge J\left(x_{m+1}, y_{m}\right)=\operatorname{dist}(A, B)\right\}$. Since $(A, B)$ satisfies the $\mathrm{WP}^{I}$-property, we conclude that

$$
\forall_{m \in \mathbb{N}}\left\{J\left(x_{m}, x_{m+1}\right) \leq J\left(y_{m-1}, y_{m}\right)\right\} .
$$


Now, we obtain

$$
\begin{aligned}
J\left(x_{m}, x_{m+1}\right) & \leq J\left(y_{m-1}, y_{m}\right) \leq \frac{\beta}{s-\beta} J\left(x_{m-1}, x_{m}\right) \\
& \leq \frac{\beta}{s-\beta} J\left(y_{m-2}, y_{m-1}\right) \leq\left(\frac{\beta}{s-\beta}\right)^{2} J\left(x_{m-2}, x_{m-1}\right) \\
& \leq\left(\frac{\beta}{s-\beta}\right)^{2} J\left(y_{m-3}, y_{m-2}\right) \leq\left(\frac{\beta}{s-\beta}\right)^{3} J\left(x_{m-3}, x_{m-2}\right) \\
& \leq \cdots \leq\left(\frac{\beta}{s-\beta}\right)^{m} J\left(y_{0}, y_{1}\right) \leq\left(\frac{\beta}{s-\beta}\right)^{m+1} J\left(x_{0}, x_{1}\right) .
\end{aligned}
$$

So, for each $m>n$ we have

$$
\begin{aligned}
J\left(x_{n}, x_{m}\right) & \leq s\left[J\left(x_{n}, x_{n+1}\right)+J\left(x_{n+1}, x_{m}\right)\right] \\
& \leq s J\left(x_{n}, x_{n+1}\right)+s\left[J\left(x_{n+1}, x_{n+2}\right)+J\left(x_{n+2}, x_{m}\right)\right] \\
& =s J\left(x_{n}, x_{n+1}\right)+s^{2} J\left(x_{n+1}, x_{n+2}\right)+s^{2} J\left(x_{n+2}, x_{m}\right) \\
& \leq \cdots \leq \sum_{k=0}^{m-(n+1)} s^{k+1} J\left(x_{n+k}, x_{n+k+1}\right) \leq \sum_{k=0}^{m-(n+1)} s^{k+1}\left(\frac{\beta}{s-\beta}\right)^{k+n+1} J\left(x_{0}, x_{1}\right) \\
& \leq\left(\frac{\beta}{s-\beta}\right)^{n} \sum_{k=0}^{m-(n+1)}\left(\frac{s \beta}{s-\beta}\right)^{k+1} J\left(x_{0}, x_{1}\right) .
\end{aligned}
$$

Since $\frac{s \beta}{s-\beta}<1$, if $n \rightarrow \infty$ in the above relation, we obtain

$$
\lim _{n \rightarrow \infty} \sup _{m>n} J\left(x_{n}, x_{m}\right)=0
$$

Similarly,

$$
\begin{aligned}
& \forall_{n>m}\left\{J\left(y_{n}, y_{m}\right) \leq \sum_{k=0}^{m-(n+1)}\left(\frac{s \beta}{s-\beta}\right) s^{k} J\left(x_{n+k}, x_{n+k+1}\right)\right. \\
& \left.\leq\left(\frac{s \beta}{s-\beta}\right)^{n+1} \sum_{k=0}^{m-(n+1)}\left(\frac{s \beta}{s-\beta}\right)^{k} J\left(x_{0}, x_{1}\right)\right\} .
\end{aligned}
$$

Thereby, $\lim _{n \rightarrow \infty} \sup _{m>n} J\left(y_{n}, y_{m}\right)=0$. From Lemma 2.11 it follows that $\left(x_{m}: m \in\{0\} \cup \mathbb{N}\right)$ and $\left(y_{m}: m \in\{0\} \cup \mathbb{N}\right)$ are Cauchy sequences in $A$ and $B$, respectively. Since $(A, B)$ is a closed pair of subsets of the complete $b$-metric space $X$, there exists $p \in A$ and $q \in B$ such that $x_{m} \rightarrow p$ and $y_{m} \rightarrow q$. Besides, since $\forall_{m \in\{0\} \cup \mathbb{N}}\left\{y_{m} \in T\left(x_{m}\right)\right\}$, by the closedness of $T$, we obtain $q \in T p$. On the other hand, since $\forall_{m \in \mathbb{N}}\left\{J\left(x_{m}, y_{m-1}\right)=\operatorname{dist}(A, B)\right\}$ and $J$ is associated with $(A, B)$, we conclude that $d(p, q)=\operatorname{dist}(A, B)$. We now have

$$
\operatorname{dist}(A, B) \leq \mathcal{D}(p, B) \leq \mathcal{D}(p, T p) \leq d(p, q)=\operatorname{dist}(A, B)
$$

that is, $\mathcal{D}(p, T p)=\operatorname{dist}(A, B)$ and so $p \in A$ is a best proximity point of the non-selfmapping $T$. 
The next results are straightforward consequences of Theorem 3.2.

Corollary 3.3 Let $X$ be a complete b-metric space (with $s \geq 1$ ). Let $(A, B)$ be a pair of nonempty closed subsets of $X$ with $A_{0} \neq \emptyset$ and such that $(A, B)$ has the WP-property. Let $T: A \rightarrow 2^{B}$ be a closed multivalued non-self-mapping which is an SK-contraction with respect to the b-metric. If $T(x) \in \mathcal{C B}(X)$ for all $x \in A$, and $T(x) \subset B_{0}$ for each $x \in A_{0}$, then $T$ has a best proximity point in $A$.

Corollary 3.4 Let $(A, B)$ be a pair of nonempty closed subsets of a complete metric space $(X, d)$ such that $A_{0} \neq \emptyset$ and $(A, B)$ satisfies the WP-property. Let $T: A \rightarrow 2^{B}$ be a closed multivalued non-self-mapping which is SK-contraction with respect to the $b$-metric, that is,

$$
\begin{aligned}
& \exists_{\alpha \in\left[0, \frac{1}{2}\right)} \forall_{x, y \in A}\left\{\left\{\frac{\zeta(\alpha)}{s} \mathcal{D}^{*}(x, T(x)) \leq d(x, y)\right\}\right. \\
& \left.\Rightarrow\left\{s \mathcal{H}(T(x), T(y)) \leq \alpha\left[\mathcal{D}^{*}(x, T x)+\mathcal{D}^{*}(y, T y)\right]\right\}\right\} .
\end{aligned}
$$

If $T(x) \in \mathcal{C B}(X)$ for all $x \in A$, and $T\left(x_{0}\right)$ is included in $B_{0}$ for each $x_{0} \in A_{0}$, then $T$ has a best proximity point in $A$.

Corollary 3.5 Let $X$ be a complete b-metric space (with $s \geq 1$ ) and let the map J $: X \times X \rightarrow$ $[0, \infty)$ be a b-generalized pseudodistance on $X$. Let $(A, B)$ be a pair of nonempty closed subsets of $X$ with $A_{0} \neq \emptyset$ and such that $(A, B)$ has the WP'-property and $J$ is associated with $(A, B)$. Let $T: A \rightarrow B$ be a continuous single-valued non-self-mapping which is an SKcontraction with respect to b-generalized pseudodistances. If $T\left(A_{0}\right) \subseteq B_{0}$, then $T$ has a best proximity point in $A$.

Corollary 3.6 Let $X$ be a complete b-metric space (with $s \geq 1$ ). Let $(A, B)$ be a pair of nonempty closed subsets of $X$ with $A_{0} \neq \emptyset$ and such that $(A, B)$ has the WP-property. Let $T: A \rightarrow B$ be a continuous single-valued non-self-mapping which is an SK-contraction with respect to the $b$-metric. If $T\left(A_{0}\right) \subseteq B_{0}$, then $T$ has a best proximity point in $A$.

Corollary 3.7 Let $X$ be a complete b-metric space (with $s \geq 1$ ). Let $A$ be a nonempty closed subset of $X$. Let $T: A \rightarrow 2^{A}$ be a closed multivalued mapping which is an SK-contraction with respect to the $b$-metric. If $T(x) \in \mathcal{C B}(X)$ for all $x \in A$, then $T$ has a fixed point.

Notice that we can omit the condition of closedness of the multivalued mapping $T$ in Corollary 3.7.

Theorem 3.8 (Compare with Theorem 1.2) Let $X$ be a complete b-metric space (with $s \geq 1)$. Let $A$ be a nonempty closed subset of $X$. Let $T: A \rightarrow 2^{A}$ be a multivalued mapping which is an SK-contraction. If $T(x) \in \mathcal{C B}(X)$ for all $x \in A$, then $T$ has a fixed point.

Proof In especial case, if $J=d$ and $A=B$ in Theorem 3.2, then we obtain a sequence $\left(x_{m}\right.$ : $m \in\{0\} \cup \mathbb{N}$ ) such that

$$
\begin{aligned}
& \forall_{m \in\{0\} \cup \mathbb{N}}\left\{x_{m+1} \in T\left(x_{m}\right)\right\}, \\
& \forall_{m \in \mathbb{N}}\left\{d\left(x_{m}, x_{m+1}\right) \leq \frac{\beta}{s-\beta} d\left(x_{m-1}, x_{m}\right)\right\} .
\end{aligned}
$$


Moreover, we proved that $x_{m} \rightarrow p \in A$. We now assert that

$$
\forall_{x \in A-\{p\}}\left\{D(p, T x) \leq \frac{\alpha}{s} D(x, T x)\right\} .
$$

Since $x_{m} \rightarrow p$ and $d$ is continuous, $d\left(x_{m}, p\right) \rightarrow 0$. So,

$$
\exists_{N \in \mathbb{N}} \forall_{m \geq N}\left\{d\left(p, x_{m}\right) \leq \frac{1}{3 s} d(p, x)\right\} .
$$

We have

$$
D\left(x_{m}, T x_{m}\right) \leq d\left(x_{m}, x_{m+1}\right) \leq s\left[d\left(x_{m}, p\right)+d\left(p, x_{m+1}\right)\right]
$$

So, $\frac{1}{s} D\left(x_{m}, T x_{m}\right) \leq d\left(x_{m}, p\right)+d\left(p, x_{m+1}\right)$. By the fact that $\zeta(\alpha) \leq 1$, for all $m>N$ we obtain

$$
\begin{aligned}
\frac{\zeta(\alpha)}{s} D\left(x_{m}, T x_{m}\right) & \leq\left[d\left(x_{m}, p\right)+d\left(p, x_{m+1}\right)\right] \\
& \leq \frac{2}{3 s} d(p, x)=\frac{1}{s} d(p, x)-\frac{1}{3 s} d(p, x) \\
& \leq \frac{1}{s} d(p, x)-d\left(p, x_{m}\right) \leq d\left(x_{m}, x\right) .
\end{aligned}
$$

Thereby,

$$
\forall_{m \geq N}\left\{s \mathcal{H}\left(T x_{m}, T x\right) \leq \alpha\left[D\left(x_{m}, T x_{m}\right)+D(x, T x)\right]\right\} \leq \alpha\left[d\left(x_{m}, x_{m+1}\right)+D(x, T x)\right]
$$

We have

$$
s D\left(x_{m+1}, T x\right) \leq s \mathcal{H}\left(T x_{m}, T x\right) \leq \alpha\left[d\left(x_{m}, x_{m+1}\right)+D(x, T x)\right]
$$

Letting $m \rightarrow \infty$, we obtain $D(p, T x) \leq \frac{\alpha}{s} D(x, T x)$, that is, (3.3) holds. We next verify that $\forall_{x \in A}\{s \mathcal{H}(T p, T x) \leq \alpha[D(p, T p)+D(x, T x)]\}$. This is trivial when $x=p$. Suppose $x \neq p$. Then for every $n \in \mathbb{N}$ there exists $z_{n} \in T x$ such that $d\left(p, z_{n}\right) \leq D(p, T x)+\frac{1}{n} d(x, p)$. So,

$$
D(x, T x) \leq d\left(x, z_{n}\right) \leq s\left[d(x, p)+d\left(p, z_{n}\right)\right]
$$

which, by (3.3), implies that

$$
\frac{1}{s} D(x, T x) \leq d(x, p)+\frac{1}{n} d(x, p)+D(p, T x) \leq d(x, p)+\frac{1}{n} d(x, p)+\frac{\alpha}{s} D(x, T x) .
$$

Thus $\frac{\zeta(\alpha)}{s} D(x, T x) \leq\left(1+\frac{1}{n}\right) d(p, x)$ for all $n \in \mathbb{N}$, from which one concludes that $\frac{\zeta(\alpha)}{s} D(x$, $T x) \leq d(p, x)$. So, $s \mathcal{H}(T p, T x) \leq \alpha[D(p, T p)+D(x, T x)]$. Hence,

$$
\begin{aligned}
D(p, T p) & =\lim _{m \rightarrow \infty} D\left(x_{m}, T p\right) \\
& \leq \lim _{m \rightarrow \infty} \mathcal{H}\left(T x_{m-1}, T p\right) \leq \lim _{m \rightarrow \infty} \frac{\alpha}{s}\left[D(p, T p)+D\left(x_{m-1}, T x_{m-1}\right)\right] \\
& \leq \frac{\alpha}{s} D(p, T p)+\lim _{m \rightarrow \infty} \frac{\alpha}{s} d\left(x_{m-1}, x_{m}\right)=\frac{\alpha}{s} D(p, T p) .
\end{aligned}
$$


Since $\frac{\alpha}{s}<1$, we must have $D(p, T p)=0$. That is, $p \in \overline{T p}=T p$ and this completes the proof of the theorem.

\section{Examples and remarks}

Now, we will present some examples illustrating the concepts having been introduced so far. First, we present an example of generalized pseudodistances in metric spaces and $b$-metric spaces, respectively.

Example 4.1 Let $X$ be a metric space ( $b$-metric space respectively) where the metric $d$ : $X \times X \rightarrow[0, \infty)$ is of the form $d(x, y)=|x-y|\left(b\right.$-metric $\left.d(x, y)=|x-y|^{2}\right), x, y \in X$. Let the closed set $E \subset X$, containing at least two different points, be arbitrary and fixed. Let $c>0$ such that $c>\delta(E)$, where $\delta(E)=\sup \{d(x, y): x, y \in E\}$ be arbitrary and fixed. Define the map $J: X \times X \rightarrow[0, \infty)$ as follows:

$$
J(x, y)= \begin{cases}d(x, y) & \text { if }\{x, y\} \cap E=\{x, y\} \\ c & \text { if }\{x, y\} \cap E \neq\{x, y\} .\end{cases}
$$

Then $J: X \times X \rightarrow[0, \infty)$ is a generalized pseudodistance on $X[36]$ (b-generalized pseudodistance on $X[29])$.

Let us illustrate Theorem 3.2 with the following example.

Example 4.2 Let $(X, d)$ be a $b$-metric space (with constant $s=2$ ), where $X=\mathbb{R}, d(x, y)=$ $|x-y|^{2}, x, y \in X$. Let $(A, B)$ be a pair of subsets of $X$, where $A=[2,3]$ and $B=[0,1]$. Let $E=[0,1] \cup\{2,3\}$ and let $J: X \times X \rightarrow[0, \infty)$ be defined by the formula

$$
J(x, y)=\left\{\begin{array}{ll}
d(x, y) & \text { if } E \cap\{x, y\}=\{x, y\}, \\
10 & \text { if } E \cap\{x, y\} \neq\{x, y\},
\end{array} \quad x, y \in X\right.
$$

It is easy to show that $J$ is a $b$-generalized pseudodistance. Assume that $T: A \rightarrow 2^{B}$ is of the form

$$
T(x)= \begin{cases}\{1\} & \text { if } x=2, \\ {\left[\frac{3}{4}, 1\right]} & \text { if } x \in(2,3) \\ \left\{\frac{1}{4}\right\} & \text { if } x=3\end{cases}
$$

I. We show that the pair $(A, B)$ has the WP -property.

Indeed, we observe that $\operatorname{dist}(A, B)=1$ and by (4.2) we obtain

$$
\begin{aligned}
& A_{0}=\{x \in A: \text { there exists } u \in B \text { such that } J(x, u)=d(A, B)\}=\{2\}, \\
& B_{0}=\{u \in B: \text { there exists } x \in B \text { such that } J(x, u)=d(A, B)\}=\{1\} .
\end{aligned}
$$

Hence, it is easy to verify that the pair $(A, B)$ has the $\mathrm{WP}^{J}$-property.

II. We see that $A$ is complete and by (4.3) we have $T\left(A_{0}\right)=T(\{2\})=\{1\} \subset B_{0}$.

III. We see that $T$ is an $S K$-contraction with respect to the b-generalized pseudodistance. 
Indeed, first we observe that by (4.2) and the definition of $E$, we calculate

$$
\mathcal{H}^{J}\left(\left[\frac{3}{4}, 1\right],\left\{\frac{1}{4}\right\}\right)=\mathcal{H}^{J}\left(\{1\},\left\{\frac{1}{4}\right\}\right)=\frac{9}{16} .
$$

Let $\alpha=\frac{181}{365}<\frac{1}{2}$ and let $x, y \in A$ be arbitrary and fixed. Additionally, we calculate

$$
\begin{aligned}
& J^{*}(2, T(2))=\frac{1}{2} J(2, T(2))-1=\frac{1}{2} J(2,\{1\})-1=-\frac{1}{2}, \\
& J^{*}(3, T(3))=\frac{1}{2} J(3, T(3))-1=\frac{1}{2} J\left(3,\left[0, \frac{1}{4}\right]\right)-1=\frac{121}{32}-1=\frac{89}{32}
\end{aligned}
$$

and

$$
\forall_{x \in(2,3)}\left\{J^{*}(x, T(x))=\frac{1}{2} J\left(x,\left[\frac{3}{4}, 1\right]\right)-1\right\}=4 \quad(\text { since } x \notin E) .
$$

Now, we consider the following cases.

Case 1 . If $x=2$ and $y=3$, then by (4.3)-(4.6) we obtain

$$
\begin{aligned}
2 \mathcal{H}^{J}(T(x), T(y)) & =2 \mathcal{H}^{J}\left(\{1\},\left\{\frac{1}{4}\right\}\right)=\frac{9}{8}=\frac{13,140}{11,680}<\frac{13,213}{11,680}=\frac{181}{365} \cdot \frac{73}{32} \\
& =\frac{181}{365}\left[-\frac{1}{2}+\frac{89}{32}\right]=\frac{181}{365}\left[J^{*}(x, T(x))+J^{*}(y, T(y))\right] .
\end{aligned}
$$

Case 2. If $x=3$ and $y=2$, then by (4.2)-(4.6) we obtain

$$
\begin{aligned}
2 \mathcal{H}^{J}(T(x), T(y)) & =2 \mathcal{H}^{J}\left(\left\{\frac{1}{4}\right\},\{1\}\right)=\frac{9}{8}=\frac{13,140}{11,680}<\frac{13,213}{11,680}=\frac{181}{365} \cdot \frac{73}{32} \\
& =\frac{181}{365}\left[\frac{89}{32}+\left(-\frac{1}{2}\right)\right]=\frac{181}{365}\left[J^{*}(x, T(x))+J^{*}(y, T(y))\right] .
\end{aligned}
$$

Case 3. If $x=2$ and $y \in(2,3)$, then by (4.3)-(4.7) we obtain

$$
\begin{aligned}
2 \mathcal{H}^{J}(T(x), T(y)) & =2 \mathcal{H}^{J}\left(\{1\},\left[\frac{3}{4}, 1\right]\right)=0 \leq \frac{181}{365}\left[-\frac{1}{2}+4\right] \\
& \leq \frac{181}{365}\left[J^{*}(x, T(x))+J^{*}(y, T(y))\right] .
\end{aligned}
$$

Case 4. If $x=(2,3)$ and $y=2$, then by (4.3)-(4.7) we obtain

$$
\begin{aligned}
2 \mathcal{H}^{J}(T(x), T(y)) & =2 \mathcal{H}^{J}\left(\left[\frac{3}{4}, 1\right],\{1\}\right)=0 \leq \frac{181}{365}\left[4+\left(-\frac{1}{2}\right)\right] \\
& \leq \frac{181}{365}\left[J^{*}(x, T(x))+J^{*}(y, T(y))\right] .
\end{aligned}
$$

Case 5. If $x=3$ and $y \in(2,3)$, then by (4.3)-(4.7) we obtain

$$
\begin{aligned}
2 \mathcal{H}^{J}(T(x), T(y)) & =2 \mathcal{H}^{J}\left(\left\{\frac{1}{4}\right\},\left[\frac{3}{4}, 1\right]\right)=\frac{9}{8}=\frac{13,140}{11,680}<\frac{13,213}{11,680}=\frac{181}{365}\left[-\frac{1}{2}+\frac{89}{32}\right] \\
& <\frac{181}{365}\left[\frac{89}{32}+4\right] \leq \frac{181}{365}\left[J^{*}(x, T(x))+J^{*}(y, T(y))\right] .
\end{aligned}
$$


Case 6. If $x \in(2,3)$ and $y=3$, then by (4.3)-(4.7) we obtain

$$
\begin{aligned}
2 \mathcal{H}^{J}(T(x), T(y)) & =2 \mathcal{H}^{J}\left(\left[\frac{3}{4}, 1\right],\left\{\frac{1}{4}\right\}\right)=\frac{9}{8}=\frac{13,140}{11,680}<\frac{13,213}{11,680}=\frac{181}{365}\left[-\frac{1}{2}+\frac{89}{32}\right] \\
& <\frac{181}{365}\left[4+\frac{89}{32}\right] \leq \frac{181}{365}\left[J^{*}(x, T(x))+J^{*}(y, T(y))\right] .
\end{aligned}
$$

Case 7. If $x, y \in(2,3)$, then by (4.3)-(4.7) we obtain

$$
\begin{aligned}
2 \mathcal{H}^{J}(T(x), T(y)) & =2 \mathcal{H}^{J}\left(\left[\frac{3}{4}, 1\right],\left[\frac{3}{4}, 1\right]\right)=0 \leq \frac{181}{365}[4+4] \\
& \leq \frac{181}{365}\left[J^{*}(x, T(x))+J^{*}(y, T(y))\right] .
\end{aligned}
$$

Now, from (4.8)-(4.14), we have (we recall $s=2$ and $\alpha=\frac{181}{365}$ )

$$
\forall_{x, y \in A}\left\{s \mathcal{H}^{J}(T(x), T(y)) \leq \alpha\left[J^{*}(x, T(x))+J^{*}(y, T(y))\right]\right\} .
$$

Hence, obviously

$$
\begin{aligned}
\exists_{\alpha=\frac{181}{365} \in\left[0, \frac{1}{2}\right)} \forall_{x, y \in A}\left\{\left\{\frac{\zeta(\alpha)}{s} J^{*}(x, T(x)) \leq J(x, y)\right\}\right. \\
\left.\quad \Rightarrow\left\{s \mathcal{H}^{J}(T(x), T(y)) \leq \alpha\left[J^{*}(x, T(x))+J^{*}(y, T(y))\right]\right\}\right\} .
\end{aligned}
$$

In consequence, the map $T$ is an SK-contraction with respect to the $b$-generalized pseudodistance.

IV. We see that all assumptions of Theorem 3.2 are satisfied.

Indeed, the map $T$ is closed, and $J$ is associated with $(A, B)$ (by $(4.2)$ and continuity of $d$ ).

$\mathrm{V}$. There exists a best proximity point of $T$.

Indeed, for $z=2$ we have $\inf \{d(z, u): u \in T(z)\}=\inf \{d(2, u): u \in\{1\}\}=1=\operatorname{dist}(A, B)$.

In conclusion, in order to compare our result with Theorem 1.1 and Theorem 1.2, we need to reformulate the definition of the SK-contraction.

Definition 4.1 Define the strictly decreasing function $\zeta:\left[0, \frac{1}{2}\right) \rightarrow\left(\frac{1}{2}, 1\right]$ by

$$
\zeta(\alpha)=1-\alpha
$$

Let $X$ be a metric space (i.e. $b$-metric space with $s=1$ ) and let $J: X \times X \rightarrow[0, \infty)$ be a $b$-generalized pseudodistance (for short: generalized pseudodistance) on $X$.

(I) Let $T: X \rightarrow X$. In this case, $J^{*}(x, T(x))=J(x, T(x))$, where $x \in X$. A single-valued self-mapping $T$ is said to be a SK-contraction with respect to generalized pseudodistance provided that

$$
\begin{aligned}
& \exists_{\alpha \in\left[0, \frac{1}{2}\right)} \forall_{x, y \in X}\{\{\zeta(\alpha) J(x, T(x)) \leq J(x, y)\} \\
& \quad \Rightarrow\{J(T(x), T(y)) \leq \alpha[J(x, T x)+J(y, T y)]\}\} .
\end{aligned}
$$


(II) Let $T: X \rightarrow C B(X)$. In this case, $J^{*}(x, T(x))=J(x, T(x))=\inf \{J(x, y): y \in T(x)\}$, where $x \in X$. A multivalued self-mapping $T$ is said to be an SK-contraction with respect to the generalized pseudodistance provided that

$$
\begin{aligned}
& \exists_{\alpha \in\left[0, \frac{1}{2}\right)} \forall_{x, y \in X}\{\{\zeta(\alpha) J(x, T(x)) \leq J(x, y)\} \\
& \left.\quad \Rightarrow\left\{\mathcal{H}^{J}(T(x), T(y)) \leq \alpha[J(x, T x)+J(y, T y)]\right\}\right\} .
\end{aligned}
$$

Now, we can formulate the following remarks:

\section{Remark 4.2}

(i) There exist SK-contractions with respect to $b$-generalized pseudodistances which are not contractions of Kannan type as introduced by Kikkawa and Suzuki [6] (see Examples 4.3 and 4.4) and not contractions as introduced by Damjanovic and Doric [7] (see Examples 4.5 and 4.6).

(ii) Theorem 3.2 is an essential generalization of Theorem 1.1 (see Examples 4.3 and 4.4).

(iii) Theorem 3.2 is an essential generalization of Theorem 1.2 (see Examples 4.5 and 4.6).

Example 4.3 Let $(X, d)$ be a metric space (i.e. $b$-metric space with constant $s=1$ ), where $X=[0,2] \subset \mathbb{R}, d(x, y)=|x-y|, x, y \in X$. Let $E=\left[0, \frac{3}{2}\right]$ and let $J: X \times X \rightarrow[0, \infty)$ be defined by the formula

$$
J(x, y)=\left\{\begin{array}{ll}
d(x, y) & \text { if } E \cap\{x, y\}=\{x, y\}, \\
4 & \text { if } E \cap\{x, y\} \neq\{x, y\},
\end{array} \quad x, y \in X\right.
$$

It is easy to show that $J$ is a $b$-generalized pseudodistance (we recall $s=1$ ). Assume that $T: X \rightarrow X$ is of the form

$$
T(x)= \begin{cases}\frac{1}{2} & \text { if } x \in\left[0, \frac{3}{2}\right] \\ 2 x-\frac{5}{2} & \text { if } x \in\left(\frac{3}{2}, 2\right]\end{cases}
$$

I. We see that $T$ is an SK-contraction.

Indeed, let $\alpha=\frac{1}{3}$ and let $x, y \in X$ be arbitrary and fixed. We consider the following cases. Case 1 . If $x, y \in\left[0, \frac{3}{2}\right]$, then by $(4.16)$ we obtain $T x=T y=\frac{1}{2}$. Hence, $J(T x, T y)=d(T x, T y)=$ $0 \leq \frac{1}{3}[J(x, T x)+J(y, T y)]$. Moreover,

$$
\zeta(\alpha) J(x, T x) \leq J(x, y) \quad \text { implies } \quad J(T x, T y) \leq \alpha[J(x, T x)+J(y, T y)]
$$

Case 2. If $x, y \in\left(\frac{3}{2}, 2\right]$, then by (4.15) and (4.16) we obtain $J(x, T x)=J(y, T y)=4$. Hence, $J(T x, T y)=d(T x, T y) \leq 1$ and hence $J(T x, T y) \leq 1<\frac{8}{3}=\frac{1}{3}[J(x, T x)+J(y, T y)]$. Moreover,

$$
\zeta(\alpha) J(x, T x) \leq J(x, y) \quad \text { implies } \quad J(T x, T y) \leq \alpha[J(x, T x)+J(y, T y)]
$$

Case 3. If $x \in\left[0, \frac{3}{2}\right], y \in\left(\frac{3}{2}, 2\right]$, then by (4.15) and (4.16) we obtain $J(x, T x)=d(x, T x) \leq$ 1 and $J(y, T y)=4$. Hence, $J(T x, T y)=d(T x, T y) \leq 1$ and so $J(T x, T y) \leq 1<\frac{4}{3}=\frac{1}{3}[0+4]=$ 
$\frac{1}{3}\left[\min \left\{J(x, T x): x \in\left[0, \frac{3}{2}\right]\right\}+J(y, T y)\right] \leq \frac{1}{3}[J(x, T x)+J(y, T y)]$. Moreover,

$$
\zeta(\alpha) J(x, T x) \leq J(x, y) \quad \text { implies } \quad J(T x, T y) \leq \alpha[J(x, T x)+J(y, T y)] .
$$

Now, by (4.17)-(4.19) and the symmetry of $J$, we obtain

$$
\begin{aligned}
& \exists_{\alpha=\frac{1}{3} \in\left[0, \frac{1}{2}\right)} \forall_{x, y \in X}\{\{\zeta(\alpha) J(x, T(x)) \leq J(x, y)\} \\
& \quad \Rightarrow\{J(T(x), T(y)) \leq \alpha[J(x, T(x))+J(y, T(y))]\}\} .
\end{aligned}
$$

In consequence, the map $T$ is an SK-contraction on $X$.

II. We see that all assumptions of Theorem 3.2 are satisfied.

Indeed, the map $T$ is a closed self-mapping which is an SK-contraction.

III. There exists a fixed point for $T$.

Indeed, for $z=0$ we have $0 \in T(\{0\})$.

Example 4.4 Let $(X, d)$ be a metric space (i.e. $b$-metric space with constant $s=1)$, where $X=[0,2] \subset \mathbb{R}, d(x, y)=|x-y|, x, y \in X$. Let $T$ be as in Example 4.3. We show that the map $T$ is not a contraction of Kannan type as introduced by Kikkawa and Suzuki [6].

Indeed, we suppose that the map $T$ satisfies (1.1). Then there exists $\alpha_{0} \in\left[0, \frac{1}{2}\right)$ such that $r_{0}=\frac{\alpha_{0}}{1-\alpha_{0}}$ and

$$
\varphi\left(r_{0}\right) d(x, T x) \leq d(x, y) \quad \text { implies } \quad d(T x, T y) \leq \alpha[d(x, T x)+d(y, T y)] .
$$

In particular, for $x_{0}=1, y_{0}=2$, by (4.15) we get $T x_{0}=\frac{1}{2}, d\left(x_{0}, T x_{0}\right)=d\left(1, \frac{1}{2}\right)=\frac{1}{2}, T y_{0}=\frac{3}{2}$, $d\left(y_{0}, T y_{0}\right)=d\left(2, \frac{3}{2}\right)=\frac{1}{2}$, and

$$
\varphi\left(r_{0}\right) d\left(x_{0}, T x_{0}\right) \leq d\left(x_{0}, T x_{0}\right)=\frac{1}{2}<1=d\left(x_{0}, y_{0}\right) .
$$

Hence, by (4.20) we obtain

$$
\begin{aligned}
1 & =d\left(\frac{1}{2}, \frac{3}{2}\right)=d\left(T x_{0}, T y_{0}\right) \leq \alpha_{0}\left[d\left(x_{0}, T x_{0}\right)+d\left(y_{0}, T y_{0}\right)\right] \\
& <\left[d\left(x_{0}, T x_{0}\right)+d\left(y_{0}, T y_{0}\right)\right]=1,
\end{aligned}
$$

which is impossible. In consequence the map $T$ is not a contraction of Kannan type [6].

Example 4.5 Let $(X, d)$ be a metric space (i.e. $b$-metric space with constant $s=1)$, where $X=[0,2] \subset \mathbb{R}, d(x, y)=|x-y|, x, y \in X$. Let $E=\left[0, \frac{3}{2}\right]$ and let $J: X \times X \rightarrow[0, \infty)$ be as in Example 4.3, i.e.

$$
J(x, y)=\left\{\begin{array}{ll}
d(x, y) & \text { if } E \cap\{x, y\}=\{x, y\}, \\
4 & \text { if } E \cap\{x, y\} \neq\{x, y\},
\end{array} \quad x, y \in X .\right.
$$

Let $T: X \rightarrow 2^{X}$ be of the form

$$
T(x)= \begin{cases}\left\{\frac{1}{2}\right\} & \text { if } x \in\left[0, \frac{3}{2}\right], \\ {\left[2 x-\frac{5}{2}, x-\frac{1}{2}\right]} & \text { if } x \in\left(\frac{3}{2}, 2\right] .\end{cases}
$$


I. We see that $T$ is a multivalued self-SK-contraction.

Indeed, let $\alpha=\frac{1}{3}$ and let $x, y \in X$ be arbitrary and fixed. We consider the following cases. Case 1. If $x, y \in\left[0, \frac{3}{2}\right]$, then by (4.22) we obtain $T x=T y=\left\{\frac{1}{2}\right\}$. Hence, from (4.21), $\mathcal{H}^{J}(T x, T y)=\mathcal{H}(T x, T y)=0 \leq \frac{1}{3}[J(x, T x)+J(y, T y)]$. Moreover,

$$
\zeta(\alpha) J(x, T x) \leq J(x, y) \quad \text { implies } \quad \mathcal{H}^{J}(T x, T y) \leq \alpha[J(x, T x)+J(y, T y)] .
$$

Case 2. If $x, y \in\left(\frac{3}{2}, 2\right]$, then by (4.22) and (4.21) we obtain $J(x, T x)=J(y, T y)=4$. Hence, $\mathcal{H}^{J}(T x, T y)=\mathcal{H}(T x, T y) \leq 1$ and hence $\mathcal{H}^{J}(T x, T y) \leq 1<\frac{4}{3}=\frac{1}{3}[J(x, T x)+J(y, T y)]$. Moreover,

$$
\zeta(\alpha) J(x, T x) \leq J(x, y) \quad \text { implies } \quad \mathcal{H}^{J}(T x, T y) \leq \alpha[J(x, T x)+J(y, T y)] .
$$

Case 3. If $x \in\left[0, \frac{3}{2}\right], y \in\left(\frac{3}{2}, 2\right]$, then by (4.22) and (4.21) we obtain $J(x, T x)=d(x, T x) \leq 1$ and $J(y, T y)=4$. Hence, $\mathcal{H}^{J}(T x, T y)=\mathcal{H}(T x, T y) \leq 1$ and hence $\mathcal{H}^{J}(T x, T y) \leq 1<\frac{4}{3}=\frac{1}{3}[0+$ $4]=\frac{1}{3}\left[\min \left\{J(x, T x): x \in\left[0, \frac{3}{2}\right]\right\}+J(y, T y)\right] \leq \frac{1}{3}[J(x, T x)+J(y, T y)]$. Moreover,

$$
\zeta(\alpha) J(x, T x) \leq J(x, y) \quad \text { implies } \quad \mathcal{H}^{J}(T x, T y) \leq \alpha[J(x, T x)+J(y, T y)] .
$$

Now by (4.23)-(4.25) and the symmetry of $J$, we obtain

$$
\begin{aligned}
& \exists_{\alpha=\frac{1}{3} \in\left[0, \frac{1}{2}\right)} \forall_{x, y \in X}\{\{\zeta(\alpha) J(x, T(x)) \leq J(x, y)\} \\
& \left.\quad \Rightarrow\left\{\mathcal{H}^{J}(T(x), T(y)) \leq \alpha[J(x, T x)+J(y, T y)]\right\}\right\} .
\end{aligned}
$$

In consequence, the map $T$ is a multivalued self-SK-contraction on $X$.

II. We see that all assumptions of Theorem 3.2 are satisfied.

Indeed, the map $T$ is a closed self-mapping which is a SK-contraction.

III. There exists a fixed point for $T$.

Indeed, for $z=0$ we have $0 \in T(\{0\})$.

Example 4.6 Let $(X, d)$ be a metric space (i.e. $b$-metric space with constant $s=1$ ), where $X=[0,2] \subset \mathbb{R}, d(x, y)=|x-y|, x, y \in X$. Let $T$ be as in Example 4.5. We show that the map $T$ is not contraction as introduced by Damjanovic and Doric [7].

Indeed, we suppose that the map $T$ satisfies (1.2). Then there exists $r_{0} \in[0,1)$ such that

$$
\varphi\left(r_{0}\right) D(x, T x) \leq d(x, y) \quad \text { implies } \quad \mathcal{H}(T x, T y) \leq r_{0} \max \{D(x, T x), D(y, T y)\} .
$$

In particular, for $x_{0}=1, y_{0}=2$, by $(4.22)$ we get $T x_{0}=\left\{\frac{1}{2}\right\}, D\left(x_{0}, T x_{0}\right)=D\left(1,\left\{\frac{1}{2}\right\}\right)=\frac{1}{2}, T y_{0}=$ $\left\{\frac{3}{2}\right\}, D\left(y_{0}, T y_{0}\right)=D\left(2,\left\{\frac{3}{2}\right\}\right)=\frac{1}{2}$ and

$$
\varphi\left(r_{0}\right) D\left(x_{0}, T x_{0}\right) \leq D\left(x_{0}, T x_{0}\right)=\frac{1}{2}<1=d\left(x_{0}, y_{0}\right) .
$$

Hence, by (4.26) we obtain

$$
1=\mathcal{H}\left(\left\{\frac{1}{2}\right\},\left\{\frac{3}{2}\right\}\right)=\mathcal{H}\left(T x_{0}, T y_{0}\right) \leq r_{0} \max \left\{D\left(x_{0}, T x_{0}\right), D\left(y_{0}, T y_{0}\right)\right\}<\frac{1}{2},
$$

which is impossible. In consequence the map $T$ is not a contraction as introduced by Damjanovic and Doric [7]. 


\section{Competing interests}

The authors declare that they have no competing interests.

\section{Authors' contributions}

The main idea of this paper was proposed by MG and RP prepared the manuscript initially and performed all the steps of the proofs in this research. All authors read and approved the final manuscript.

\section{Author details}

'Department of Mathematics, Ayatollah Boroujerdi University, Boroujerd, Iran. ${ }^{2}$ Department of Nonlinear Analysis, University of Łódź, Banacha 22, Łódź, 90-238, Poland.

\section{Received: 17 October 2014 Accepted: 19 March 2015 Published online: 09 April 2015}

\section{References}

1. Khamsi, MA, Kirk, WA: An Introduction to Metric Spaces and Fixed Point Theory. Pure and Applied Mathematics. Wiley-Interscience, New York (2001)

2. Kannan, R: Some results on fixed points. Am. Math. Mon. 76(4), 405-408 (1969)

3. Reich, S: Kannan's fixed point theorem. Boll. Unione Mat. Ital. 4, 1-11 (1972)

4. Reich, S: Some remarks concerning contraction mappings. Can. Math. Bull. 14, 121-124 (1971)

5. Reich, S: Fixed points of contractive functions. Boll. Unione Mat. Ital. 5, 26-42 (1972)

6. Kikkawa, M, Suzuki, T: Some similarity between contractions and Kannan mappings. Fixed Point Theory Appl. 2010, Article ID 649749 (2010)

7. Damjanovic, B, Doric, D: Multivalued generalizations of the Kannan fixed point theorem. Filomat 25, 125-131 (2011)

8. Kirk, WA, Reich, S, Veeramani, P: Proximinal retracts and best proximity pair theorems. Numer. Funct. Anal. Optim. 24, 851-862 (2003)

9. Abkar, A, Gabeleh, M: The existence of best proximity points for multivalued non-self-mappings. Rev. R. Acad. Cienc. Exactas Fís. Nat., Ser. A Mat. 107, 319-325 (2013)

10. Gabeleh, M: Best proximity points: global minimization of multivalued non-self mappings. Optim. Lett. 8, 1101-1112 (2014)

11. Gabeleh, M: Best proximity point theorems for single- and set-valued non-self mappings. Acta Math. Sci., Ser. B 34, 1661-1669 (2014)

12. Wlodarczyk, K, Plebaniak, R, Banach, A: Best proximity points for cyclic and noncyclic set-valued relatively quasi-asymptotic contractions in uniform spaces. Nonlinear Anal. 70, 3332-3342 (2009)

13. Wlodarczyk, K, Plebaniak, R, Banach, A: Erratum to: 'Best proximity points for cyclic and noncyclic set-valued relatively quasi-asymptotic contractions in uniform spaces'. Nonlinear Anal. 71, 3583-3586 (2009)

14. Sankar Raj, V: A best proximity point theorem for weakly contractive non-self-mappings. Nonlinear Anal. 74 4804-4808 (2011)

15. Gabeleh, M: Global optimal solutions of non-self mappings. Sci. Bull. 'Politeh.' Univ. Buchar., Ser. A, Appl. Math. Phys. 75, 67-74 (2013)

16. Abkar, A, Gabeleh, M: Global optimal solutions of noncyclic mappings in metric spaces. J. Optim. Theory Appl. 153 298-305 (2012)

17. Gabeleh, M, Shahzad, N: Existence and uniqueness of a solution for some nonlinear programming problems. Mediterr. J. Math. 12, 133-146 (2015). doi:10.1007/s00009-013-0380-z

18. Czerwik, S: Nonlinear set-valued contraction mappings in b-metric spaces. Atti Semin. Mat. Fis. Univ. Modena 46 263-276 (1998)

19. Kirk, WA, Shahzad, N: Fixed Point Theory in Distance Spaces. Springer, New York (2014)

20. Eldred, AA, Veeramani, P: Existence and convergence of best proximity points. J. Math. Anal. Appl. 323(2), 1001-1006 (2006)

21. Al-Thagafi, MA, Shahzad, N: Convergence and existence results for best proximity points. Nonlinear Anal. 70(10), 3665-3671 (2009)

22. Ali, MU, Kamran, T, Shahzad, N: Best proximity point for $\alpha$ - $\psi$-proximal contractive multimaps. Abstr. Appl. Anal. 2014 Article ID 181598 (2014)

23. Karpinar, E, Raju Kosuru, GS, Tas, K: Best proximity theorems for Reich type cyclic orbital and Reich type Meir-Keeler contraction maps. J. Nonlinear Anal. Optim. 5(1), 1-10 (2014)

24. Karpinar, E, Petrusel, G, Tas, K: Best proximity point theorems for KT-types cyclic orbital contraction mappings. Fixed Point Theory 13(2), 537-545 (2012)

25. Sadiq Basha, S, Shahzad, N: Best proximity point theorems for generalized proximal contractions. Fixed Point Theory Appl. 2012, 42 (2012)

26. Al-Thagafi, MA, Shahzad, N: Best proximity pairs and equilibrium pairs for Kakutani multimaps. Nonlinear Anal. 70(3), 1209-1216 (2009)

27. Suzuki, T, Kikkawa, M, Vetro, C: The existence of best proximity points in metric spaces with the property UC. Nonlinear Anal. 71(7-8), 2918-2925 (2009)

28. Shukla, S: Partial b-metric spaces and fixed point theorems. Mediterr. J. Math. 11, 703-711 (2014)

29. Plebaniak, R: On best proximity points for set-valued contractions of Nadler type with respect to $b$-generalized pseudodistances in b-metric spaces. Fixed Point Theory Appl. 2014, 39 (2014)

30. Kada, O, Suzuki, T, Takahashi, W: Nonconvex minimization theorems and fixed point theorems in complete metric spaces. Math. Jpn. 44(2), 381-391 (1996)

31. Tataru, D: Viscosity solutions of Hamilton-Jacobi equations with unbounded nonlinear terms. J. Math. Anal. Appl. 163(2), 345-392 (1992)

32. Suzuki, T: Generalized distance and existence theorems in complete metric spaces. J. Math. Anal. Appl. 253(2), 440-458 (2001)

33. Lin, L-J, Du, W-S: Ekeland's variational principle, minimax theorems and existence of nonconvex equilibria in complete metric spaces. J. Math. Anal. Appl. 323(1), 360-370 (2006) 
34. Valyi, I: A general maximality principle and a fixed point theorem in uniform space. Period. Math. Hung. 16(2), 127-134 (1985)

35. Wlodarczyk, K, Plebaniak, R: Maximality principle and general results of Ekeland and Caristi types without lower semicontinuity assumptions in cone uniform spaces with generalized pseudodistances. Fixed Point Theory Appl. 2010, Article ID 175453 (2010)

36. Wlodarczyk, K, Plebaniak, R: Contractions of Banach, Tarafdar, Meir-Keeler, Ćirić-Jachymski-Matkowski and Suzuki types and fixed points in uniform spaces with generalized pseudodistances. J. Math. Anal. Appl. 404(2), 338-350 (2013)

Submit your manuscript to a SpringerOpen ${ }^{\circ}$ journal and benefit from:

- Convenient online submission

Rigorous peer review

- Immediate publication on acceptance

- Open access: articles freely available online

- High visibility within the field

- Retaining the copyright to your article 